\title{
Subject Index Vol. 7, 1996
}

X-Acetylcamitine 147 Acetylcholinesterase 147

- activity 111 Acute phase response 201 Administration, cutaneous 47 Age 251 Ag(e)ing 95,128

- brain 147 Agnosia 23 Albumin 256 Algorithm 324 Alurninium 1 Alzheimer's disease 1,10,15,

27,35,47,59,63,69,76, 82,86,95, 111,121,128, 135, 169, 177, 182,201, 210,215,226,233,239, 246,251,256,260,267, 273,279,293,314,331,

336,343 Amyloid P component, serum levels 256

- $\quad$ precursor protein 1,82

p-Amyloid deposits 86

Antagonism 331

Antipsychotic agents 142

Aphasia 23

Apolipoprotein E 169,251,

273,336 Apraxia 23 Aprosody 59 Astrocytes 128 Atrophy 15 Autoimmunity 91

Ballistocardiography 279 Behavio(u)ral disorders) 142, 155

- neuroanatomy 239

Binswanger's disease 53 Brain mapping 63

- volume 304

Calcium homeostasis 161

Case finding 336

Cerebral glucose metabolism 239

- ischemia 161

Cerebrospinal fluid 111,267,273

Choline acetyltransferase 233

Cholinesterase inhibitors 260

Cluster analysis 215

Clustering 121

Cognitive decline 35,169 Community studies 336 Computed tomography 27,76 
Confusion 142 Cortical areas 210

- dementia 23

- neuropathology 304 Cortico-cortical projections 86 Criteria 324

Degenerated neurites 233 Delirium 142 Delusion 142 Dementia 53, 69, 104,142,

207,221,226,279,343 -, family history 35 -, frontal lobe type 155 Depression, age-associated

memory impairment 182 Diagnosis 23

Differential diagnosis 324,331 Discriminant analysis 314 Donepezil 293 Down syndrome 69,82,135,

221,288

Elderly 142,169,279 Electroencephalogram 69

- coherence 314

- $\quad$ power 314 Electroencephalography 27 Endothelial cell 91 Epidemiology 336

Frontal cortex 128

- lobe 304

- $\quad$ - degeneration 27,128

- - dementia 267,273 Frontotemporal dementia 155

Glial fibrillary acidic protein 267 Glucocorticoids 201 Glutamate 161 Growth hormone 288

- hormone-releasing hormone

288

Hippocampus 15,76,182,226 5-HT system 196 5-HTIA 196 5-HT2 196

Immune reaction 1 Inflammation 201 Interactional therapy 207 Interleukin-2 343 Isoforms 273 Isolated amnesia 104 
Lecithin 260 Leupeptin 233 Limbic system 226 Linear measures 15 Longitudinal analysis 35 Long-term treatment 111

Magnetic resonance imaging

10,15,104 Man 187

Medial temporal lobe 15,304 Membrane fluidity 210 Memory deficit 76

- impairment 182

Mild cognitive impairment 314 Monkey 187 Morphometry 135 Movements 279 mRNA 82

Multi-infarct dementia 41,59 Multivariate analysis 215 Muscarinic receptors 187

Natural killer cell 343 Neurofibrillary changes 135

- tangles 177

Neuroimaging 10

Neuroleptics 142

Neuropathological variables 215

Neuropeptide Y 233

Neuropil threads 177

Neuroprotection 161

Nicotine 47

NMDA receptor density 210 Noncognitive symptoms 207 Normal tau protein 95

Oxygen radicals 161

Paired helical filaments 177

- $\quad$ - -, tau protein 95

Parkinson*s disease 53

$\left[{ }^{3} \mathrm{H}\right]$-Paroxetine 196

Pharmacokinetics 111

Phosphorylation 177

Photic stimulation 69

Plaques 121, 135

Population study 221

Bositron emission tomography

104,187,239 Power spectrum 69 Prevalence 221 Principal components analysis

215 Progression 246 
Prospective study 169 Protease inhibitor 233 Psychotherapy 207 Pulsatility index 41 Pyridostigmine 288

Quantitative electroencephalography 314

Randomized clinical trials 260 Remoxipride 142 Respiration 279

Screening tests 336

Selective acetylcholinesterase inhibitor 293

Senile dementia of Alzheimer type 41

Sigma sites 187

Skin fibroblast 82

Sleep 279

Somatosensory evoked potentials 53

Somatostatin 233

Spatial patterns 86

SPECT neuropsychology 155

Spontaneous speech impairment 35

Subcortical dementia 23

Subtypes 215

Synapses 128,226

Synaptic disconnection 86

- plasma membranes 147

Synaptophysin 128

Synergy 331

Tacrine 111,260 Tangles 121 Tau 1,177

Temporal amygdala 10 Total protein 256 Transcranial Doppler sonography 41 Transdermal application 47

Vascular dementia 196,267,

273 Verbal fluency 246 Visual dysfunction 63

- $\quad$ evoked potentials 53,63

Xanomeline 187 